\title{
Strange quark polarization of the nucleon: A parameter-independent prediction of the chiral potential model
}

\author{
X.B. Chen ${ }^{a, b}$, X.S. Chen ${ }^{a, c}$, Amand Faessler $^{c}$, Th. Gutsche ${ }^{c}$, F. Wang ${ }^{a}$ \\ ${ }^{a}$ Department of Physics and Center for Theoretical Physics, Nanjing University, Nanjing 210093, China \\ ${ }^{b}$ Department of Physics, Changsha Institute of Electricity, Changsha 410077, China \\ ${ }^{c}$ Institut für Theoretishe Physik, Universität Tübingen, Auf der Morgenstelle 14, D-72076 Tübingen, Germany
}

(November 14, 2018)

\begin{abstract}
We perform a one-loop calculation of the strange quark polarization $(\Delta s)$ of the nucleon in a SU(3) chiral potential model. We find that if the intermediate excited quark states are summed over in a proper way, i.e., summed up to a given energy instead of given radial and orbital quantum numbers, $\Delta s$ turns out to be almost independent of all the model parameters: quark masses, scalar- and vectorpotential strengths. The contribution from the quark-antiquark pair creation and annihilation " $Z$ " diagrams is found to be significant. Our numerical results agree quite reasonably with experiments and lattice QCD calculations.
\end{abstract}

PACS numbers: 12.39.Ki, 12.39.Fe, 12.39.Pn, 13.88.+e

The intrinsic strangeness content of the nucleon is a key ingredient to understand the structure and dynamics inside baryons. While the experimental investigation of the nucleon spin structure [1] clearly indicates that a strange quark sea exists and is also polarized relative to the nucleon spin, the successes of the naive spin-flavor $\mathrm{SU}(6)$ valence quark model in various aspects suggest that the strangeness content should belong to higher order effects for the nucleon. The SU(3) flavor chiral quark model, which couples light quarks to octet pseudoscalar mesons by the requirement of chiral symmetry, provides a natural mechanism for such a perturbative picture: at zeroth order the ground state octet baryons are described by a $\mathrm{SU}(6)$ wave function of three valence quarks, and at second order in the quark-meson coupling sea quarks can be generated by emitting a meson from the valence quark. For example, in the nucleon the strange quark can be generated by emitting a $K^{+}$from the $u$ quark or by emitting a $K^{0}$ from the $d$ quark (Fig. 1), and hence strange quarks can contribute to the nucleon spin structure.

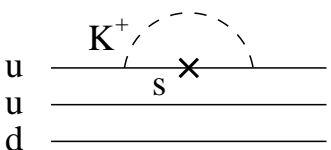

A

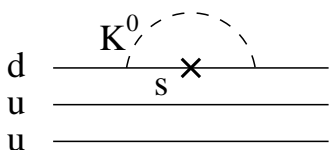

B
FIG. 1. Strange quark contribution to the nucleon spin; a cross on the quark line denotes the axial vector vertex $\gamma^{3} \gamma^{5}$.

In this paper we will adopt the standard perturbation theory to calculate the strange quark polarization of the nucleon in the framework of a $\mathrm{SU}(3)$ chiral potential model. As will be shown, up to second order the diagrams of Fig. 1 are the only contributions. Hence, a quantitative determination of the strange quark polarization is a clean test for the interaction picture of the chiral quark model, which allows in this case only admixtures of $K$ mesons. On the other hand the $u$ or $d$ quark polarizations obtain contributions from many other diagrams and therefore depend on many more model parameters.

To set up our calculation scheme, we first define the effective Lagrangian for the $\mathrm{SU}(3)$ chiral potential model:

$$
\begin{aligned}
\mathcal{L}= & \bar{\psi}\left[i \not \partial-S(r)-\gamma^{0} V(r)\right] \psi- \\
& \frac{1}{2 F_{\pi}} \bar{\psi}\left[S(r)\left(\sigma+i \gamma^{5} \lambda^{i} \phi_{i}\right)+\left(\sigma+i \gamma^{5} \lambda^{i} \phi_{i}\right) S(r)\right] \psi+ \\
& \frac{1}{2}\left(\partial_{\mu} \sigma\right)^{2}+\frac{1}{2}\left(\partial_{\mu} \phi_{i}\right)^{2}-\frac{1}{2} m_{\sigma}^{2} \sigma^{2}-\frac{1}{2} m_{i}^{2} \phi_{i}^{2}
\end{aligned}
$$

The model Lagrangian is derived from the $\sigma$ model in which meson fields are introduced to restore chiral symmetry [3]. $\psi$ is the quark field with flavor and color indices implied; the scalar term $S(r)=c r+m$ represents the the linear scalar confinement potential $\mathrm{cr}$ and the quark mass matrix $m ; V(r)=-\alpha / r$ is the Coulomb type vector potential and $F_{\pi}=93 \mathrm{MeV}$ is the pion decay constant. $\sigma$ and $\phi_{i}$ ( $i$ runs from 1 to 8 ) are the scalar and pseudoscalar meson fields, respectively and $\lambda_{i}$ are the Gell-Mann matrices. The quark-meson interaction term of Eq.(1) is symmetrized since the mass matrix $m$ does not commute with all $\lambda_{i}$ for different quark masses.

The zeroth order quark Hamiltonian is set up as

$$
H_{q}=\int d^{3} x \psi^{\dagger}\left[\vec{\alpha} \cdot \frac{1}{i} \vec{\partial}+\beta S(r)+V(r)\right] \psi
$$

It has discrete eigenstates which are obtained by numerical solution of the Dirac equation with a scalar and vector field [2]. We write the solution as:

$$
\psi(x)=\sum_{\alpha} u_{\alpha}(x) a_{\alpha}+\sum_{\beta} v_{\beta}(x) b_{\beta}^{\dagger} .
$$

Eq. (3) forms the basis of our unperturbed wave functions, where quarks are bound permanently by the confinement potential which is included in $H_{q}$. From Eq. (3) we can construct the quark propagator: 


$$
\begin{aligned}
D\left(x_{1}, x_{2}\right) \equiv & \left\langle 0\left|T\left\{\psi\left(x_{1}\right), \bar{\psi}\left(x_{2}\right)\right\}\right| 0\right\rangle \\
= & \theta\left(t_{1}-t_{2}\right) \sum_{\alpha} u_{\alpha}\left(x_{1}\right) \overline{u_{\alpha}}\left(x_{2}\right)- \\
& \theta\left(t_{2}-t_{1}\right) \sum_{\beta} v_{\beta}\left(x_{1}\right) \overline{v_{\beta}}\left(x_{2}\right),
\end{aligned}
$$

The meson propagator given by Eq. (11) is the free one:

$$
\begin{aligned}
\Delta_{i j}\left(x_{1}, x_{2}\right) & \equiv\left\langle 0\left|T\left\{\phi_{i}\left(x_{1}\right), \phi_{j}\left(x_{2}\right)\right\}\right| 0\right\rangle \\
& =\frac{i}{(2 \pi)^{4}} \int d^{4} q \frac{\delta_{i j} e^{-i q \cdot\left(x_{1}-x_{2}\right)}}{q^{2}-m_{i}^{2}+i \epsilon} .
\end{aligned}
$$

Given the unperturbed basis we can construct any physical quantity up to a desired order in the quarkmeson interaction. In the following we are studying the quark contribution of flavor $q(q=u, d, s)$ to the nucleon spin which is defined through

$$
\Delta q=\frac{\left\langle N\left|\int d^{3} x \bar{\psi}_{q} \gamma^{3} \gamma^{5} \psi_{q}\right| N\right\rangle}{\langle N \mid N\rangle}
$$

At zeroth order $H_{q}$ gives the usual SU(6) three-quark states for the nucleon with the single quark wave function $u_{\alpha}$ in the ground state. The zeroth order diagram for the numerator of Eq. (6) is indicated in Fig. 2A, and the denominator by the diagram of Fig. $3 \mathrm{~A}$, which is simply unity. Clearly, strange quarks do not contribute at this order.

The corresponding Feynman diagrams which contribute to $\Delta s$ up to second order are shown in Figs. 2 and 3. The denominator $\langle N \mid N\rangle$ can be denoted as $\left(1+\right.$ const. $\left./ F_{\pi}^{2}\right)$, which can be expanded $\left(1-\right.$ const. $/ F_{\pi}^{2}+$ $\ldots)$ and has then to be multiplied with the numerator $\left\langle N\left|\int d^{3} x \bar{\psi} \gamma^{3} \gamma^{5} \psi\right| N\right\rangle$. If finally, only terms of order $1 / F_{\pi}^{2}$ are kept in the product of the normalization and the matrix element of the spin, this has no effect on $\Delta s$, since already the lowest order admixture of $s$ quark is proportional to $1 / F_{\pi}^{2}$.

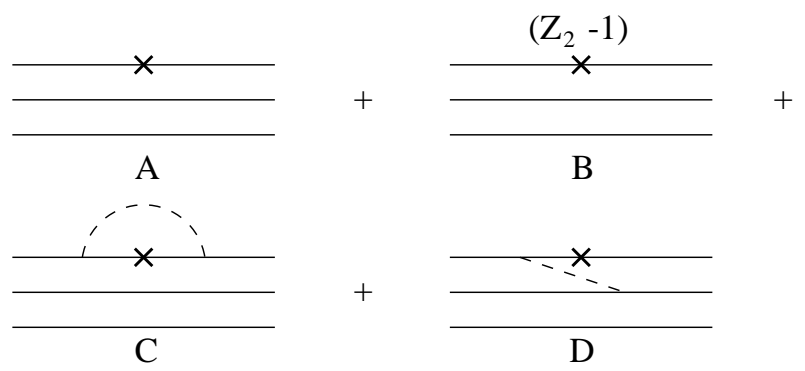

FIG. 2. Feynman diagrams for the matrix element $\left\langle N\left|\int d^{3} x \bar{\psi} \gamma^{3} \gamma^{5} \psi\right| N\right\rangle$ up to second order; A is of the zeroth order, $\mathrm{B}$ is the renormalization counter term, $\mathrm{C}$ and $\mathrm{D}$ are vertex and exchange diagrams respectively. The meson line in $\mathrm{C}$ can be a $\pi$ and $\eta$ (while the intermediate quark is $u$ or $d$ ), or a $K$ (while the intermediate quark is $s$ ); the meson line in D can only be a $\pi$ or $\eta$.

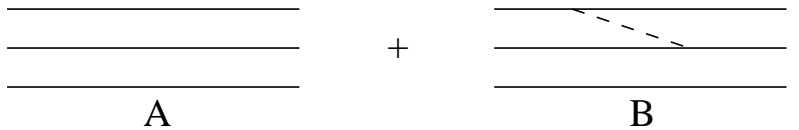

FIG. 3. Feynman diagrams for the normalization $\langle N \mid N\rangle$ up to second order; A is of the zeroth order which is simply unity, $\mathrm{B}$ is the meson exchange diagram. The meson line in $\mathrm{B}$ is a $\pi$ or $\eta$.

In the Lagrangian of Eq. (11) the main effect of the nonperturbative quark-gluon interaction is supposed to be included by the scalar and vector potentials. In principle we can also include a residual perturbative gluon piece. This will introduce further modifications on $\Delta u$ and $\Delta d$. However since the perturbative quark-gluon interaction is diagonal in flavor space, it cannot generate strange quark admixtures in second order for the nucleon.

Now we are in a good position to calculate $\Delta s$ for the nucleon: up to second order the only diagrams we need to consider are the subset of the diagrams of Fig. 2, which are given in Fig. 1. For the evaluation, we first give the explicit form for $u_{\alpha}(x)$ and $v_{\beta}(x)$ with: $u_{\alpha}(x)=e^{-i E_{\alpha} t} u_{\alpha}(\vec{x}) \tau_{\alpha}, v_{\beta}(x)=e^{i E_{\beta} t} v_{\beta}(\vec{x}) \tau_{\beta}$, where $\tau$ is the flavor wavefunction and the spatial wavefunction is:

$$
u_{\alpha}(\vec{x})=\left(\begin{array}{c}
g_{n j l} \\
-i \vec{\sigma} \cdot \overrightarrow{\vec{r}} f_{n j l}
\end{array}\right) Y_{j l}^{m}=\left(\begin{array}{c}
g_{n j l} Y_{j l}^{m} \\
i f_{n j l} Y_{j l^{\prime}}^{m}
\end{array}\right)
$$

where $g$ and $f$ are real functions, $n$ is the radial quantum number, and $Y_{j l}^{m}(\hat{\vec{r}})$ are the vector spherical harmonics. The second equality of Eq.(17) follows from $\vec{\sigma} \cdot \hat{\vec{r}} Y_{j l}^{m}=$ $-Y_{j l^{\prime}}^{m}$ with $l^{\prime}=2 j-l$. For computational convenience, we will use exactly the same form for $v_{\beta}(x)$. Since for the antiquark solution the lower component is the large component, for $v_{\beta}(x) l$ is actually the orbital quantum number of the small component, and $\left|E_{j=l+1 / 2}\right|>\left|E_{j=l-1 / 2}\right|$. Thus for the antiquarks the sequence is inversed.

Denoting the initial and final quark states as $u_{i}$ and $u_{f}$ respectively, the contribution of the diagrams of Fig. 1 is:

$$
\begin{aligned}
\delta s= & \frac{1}{F_{\pi}^{2}} \int d^{3} x d^{4} x_{1} d^{4} x_{2} \Delta\left(x_{2}, x_{1}\right) \bar{u}_{f}\left(x_{2}\right) S\left(r_{2}\right) \gamma^{5} \lambda^{i} \times \\
& D\left(x_{2}, x\right) \gamma^{3} \gamma^{5} D\left(x, x_{1}\right) S\left(r_{1}\right) \gamma^{5} \lambda^{i} u_{i}\left(x_{1}\right) .
\end{aligned}
$$

Here we use $\delta s$ to indicate that it is only the contribution from a single quark state. Inserting the explicit expressions for the propagators, we get

$$
\begin{aligned}
\delta s= & \frac{1}{F_{\pi}^{2}} \int d^{4} x_{1} d^{4} x_{2} \bar{u}_{f}\left(x_{2}\right) S\left(r_{2}\right) \gamma^{5} \lambda^{i} \times \\
& {\left[\theta\left(t_{2}-t\right) \theta\left(t-t_{1}\right) \sum_{\alpha \alpha^{\prime}} u_{\alpha}\left(x_{2}\right) \Delta_{\alpha \alpha^{\prime}} \bar{u}_{\alpha^{\prime}}\left(x_{1}\right)+\right.}
\end{aligned}
$$




$$
\begin{aligned}
& \theta\left(t_{1}-t\right) \theta\left(t-t_{2}\right) \sum_{\beta \beta^{\prime}} v_{\beta}\left(x_{2}\right) \Delta_{\beta \beta^{\prime}} \bar{v}_{\beta^{\prime}}\left(x_{1}\right)- \\
& \theta\left(t_{2}-t\right) \theta\left(t_{1}-t\right) \sum_{\alpha \beta^{\prime}} u_{\alpha}\left(x_{2}\right) \Delta_{\alpha \beta^{\prime}} \bar{v}_{\beta^{\prime}}\left(x_{1}\right)- \\
& \left.\theta\left(t-t_{2}\right) \theta\left(t-t_{1}\right) \sum_{\beta \alpha^{\prime}} v_{\beta}\left(x_{2}\right) \Delta_{\beta \alpha^{\prime}} \bar{u}_{\alpha^{\prime}}\left(x_{1}\right)\right] \times \\
& S\left(r_{1}\right) \gamma^{5} \lambda^{i} u_{i}\left(x_{1}\right) \frac{i}{(2 \pi)^{4}} \int d^{4} q \frac{\delta_{i j} e^{-i q \cdot\left(x_{1}-x_{2}\right)}}{q^{2}-m_{i}^{2}+i \epsilon} .
\end{aligned}
$$

where $\Delta_{\alpha \alpha^{\prime}}=\int d^{3} x \bar{u}_{\alpha} \gamma^{3} \gamma^{5} u_{\alpha^{\prime}}$, and similarly for $\Delta_{\beta \beta^{\prime}}$ etc. The four time-ordered terms in Eq.(9) correspond to the time-ordered diagrams of Fig. 4.
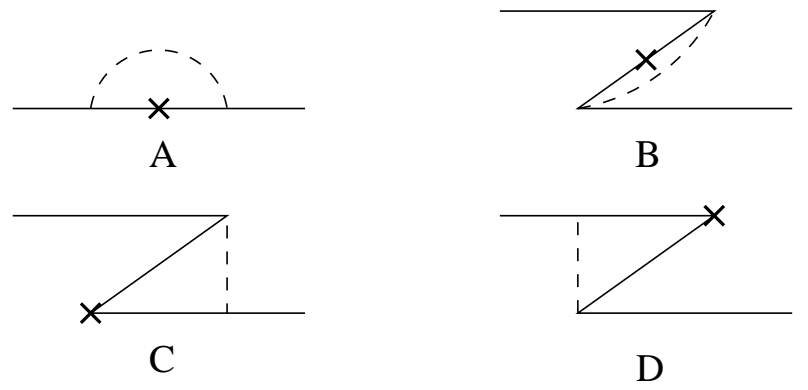

FIG. 4. Time-ordered diagrams of Fig. 1; A is the positive-energy state contribution; $\mathrm{B}$ is the negative-energy state contribution; $\mathrm{C}$ and $\mathrm{D}$ are the quark-antiquark pair creation and annihilation " $Z$ " diagrams.

We omit here the details for calculating $\delta s$ of Eq. (9). The integrals of Eq. (9) can be reduced analytically to radial integrations at the vertex points $\left(r_{1}\right.$ and $\left.r_{2}\right)$ and of loop momentum $|\vec{q}|$. The the remaining integrations are carried out numerically. $\Delta s$ for the whole nucleon is just $\delta s$ times a spin-isospin factor which can be straightforwardly calculated to be 2 .

In Table I we list our model parameters. Since $F_{\pi}=$ $93 \mathrm{MeV}$ and $m_{K}=495 \mathrm{Mev}$ are fixed by experiment, our model contains four free parameters: the two quark masses $m_{u, d}, m_{s}$ and the two strength constants of the scalar and vector potential denoted by $c$ and $\alpha$. The parameter $\alpha$ is fixed by the long-wavelength, transverse fluctuations of the QCD based static-source flux-tube picture [4.5]. It was obtained to be 0.26 in [6] and 0.30 in [7], while a much larger value of about 0.52 was used by the Cornell group [8]. Recent lattice calculation [9] got a value around 0.32 in the quenched approximation, and suggested that relaxing the quenched approximation may lead to $\alpha \sim 0.40$. Quark masses and confinement strength are rather uncertain quantities. To study the variation of $\Delta s$ over all the parameters, we choose in our calculation four different sets of parameters, including both current and constituent quark masses.

We study very different parameters because they often vary significantly from one model to another. For this model one possible choice of parameters to produce the correct nucleon mass, $g_{A}$, etc. is given in Ref. [10].

Table II gives the numerical results of $\Delta s$ for the first two sets of parameters. The intermediate quark/antiquark states are summed over up to a radial quantum number of $n=8$ and total angular momentum $j=17 / 2$. We also list the intermediate results with the summation including states up to $n=6$ and $j=11 / 2$. The contributions from the four time-ordered diagrams in Fig. 4 are given separately. We note significant contributions from Fig. 4C and Fig. 4D, in which a quarkantiquark pair is created or annihilated by the axial vector current; these processes are usually referred to as the " $Z$ " diagrams. On the other hand the diagram of Fig. $4 \mathrm{~B}$ gives a fairly large positive contribution, therefore if the " $Z$ " diagrams are neglected we would incorrectly conclude that $\Delta s$ in the nucleon is positive.

From Table II one would conclude that a stronger confinement also gives a larger $\Delta s$. This is due to the coupling of the meson field to the quark field which is proportional to the effective quark mass $S(r)=c r+m$. However, to compare with the energy scale in the lattice QCD calculation of $\Delta s$, we should sum the excited states up to a given energy instead of given radial and orbital quantum number. The resummed $\Delta s$ according to energy are given in Fig. 5. Since the quark states are discrete, we get plateaus in Fig. 5 at the energies where no new states emerge.

TABLE I. Model parameters

\begin{tabular}{ccccc}
\hline \hline $\begin{array}{c}\text { para. } \\
\text { set }\end{array}$ & $\begin{array}{c}m_{u, d} \\
{[\mathrm{MeV}]}\end{array}$ & $\begin{array}{c}m_{s} \\
{[\mathrm{MeV}]}\end{array}$ & $\alpha$ & $\begin{array}{c}c \\
{\left[\mathrm{GeV}^{2}\right]}\end{array}$ \\
\hline 1 & 10 & 150 & 0.26 & 0.11 \\
2 & 10 & 150 & 0.26 & 0.16 \\
3 & 300 & 500 & 0.26 & 0.11 \\
4 & 10 & 150 & 0.50 & 0.18 \\
\hline \hline
\end{tabular}

TABLE II. Numerical results for $\Delta s$ by summing the intermediate quark/antiquark states up to given radial and orbital quantum numbers.

\begin{tabular}{cccccccc}
\hline \hline$n$ & $j$ & set & Fig. 4A & Fig. 4B & Fig. 4C & Fig. 4D & sum \\
\hline 6 & $11 / 2$ & 1 & -.0212 & +.0829 & -.0844 & -.0844 & -.1070 \\
& & 2 & -.0340 & +.1238 & -.1255 & -.1255 & -.1613 \\
\hline 8 & $17 / 2$ & 1 & -.0220 & +.0964 & -.1109 & -.1109 & -.1475 \\
& & 2 & -.0345 & +.1445 & -.1655 & -.1655 & -.2211 \\
\hline \hline
\end{tabular}

TABLE III. Numerical results for $\Delta s$ by summing over the intermediate quark/antiquark states up to the energy $1.7 \mathrm{GeV}$.

\begin{tabular}{cccccc}
\hline \hline para. set & Fig. 4A & Fig. 4B & Fig. 4C & Fig. 4D & sum \\
\hline 1 & -.0228 & +.0785 & -.0742 & -.0742 & -.0927 \\
2 & -.0365 & +.0958 & -.0805 & -.0805 & -.1017 \\
3 & -.0464 & +.1538 & -.1046 & -.1046 & -.1018 \\
4 & -.0400 & +.0834 & -.0636 & -.0636 & -.0838 \\
\hline \hline
\end{tabular}


Since the strange axial current is a non-conserved composite operator, it has divergent matrix element (as is seen in Fig. 5), and therefore must be renormalized. Analogous to the lattice renormalization, we cut the quark intermediate states at an energy of $1.7 \mathrm{GeV}$, which is roughly the inverse of the lattice spacing in the lattice calculation of $\Delta s\left(a^{-1}=1.74 \mathrm{GeV}\right.$ in [14]). The "renormalized" results are given in Table III. (In principle, we can also do renormalization by imposing a cutoff on the meson momentum, such as using the Pauli-Villars regulator $\left(q^{2}-\Lambda^{2}\right)^{-1}$. But then the quark intermediate states have to be summed up to convergence. In practice this is not workable. An illustration with $\Lambda=1.7 \mathrm{GeV}$ is given in Fig. 5.)

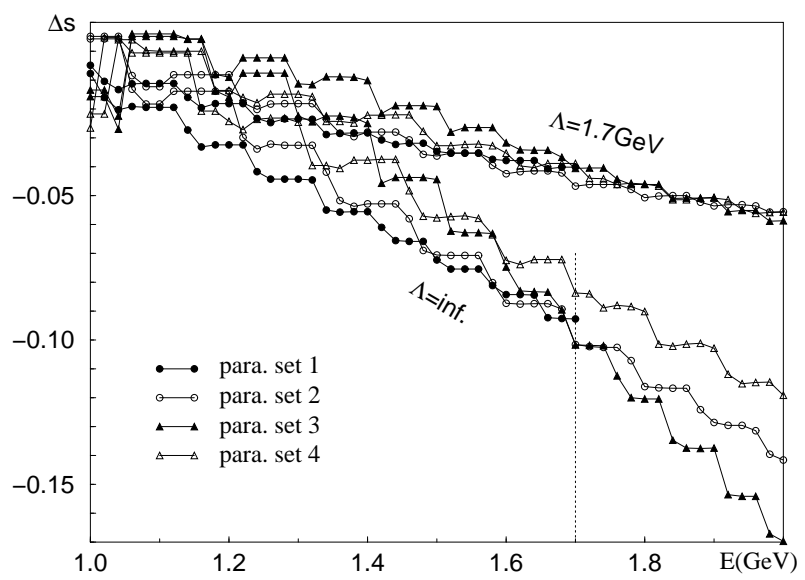

FIG. 5. plot of $\Delta s$ as a function of the maximal energy up to which the intermediate states are summed.

We note a very interesting phenomena in Fig. 5: the result for $\Delta s$ summed up to a given energy is rather robust against the variation of all the parameters. The insensitivity is especially impressive compared to the huge variation of $m$ and $c$. Table III shows that the " $Z$ " diagram's contribution is still significant.

The insensitivity of $\Delta s$ on the parameter sets can be attributed to the fact that the increase of $m$ and $c$ (see Eq. (11)) enhances the quark-meson coupling and moves up the single quark state energy. Thus the contribution from a single quark state increases due to the stronger coupling but less states are accessible to be summed over up to a given energy. Similarly, the increase of $\alpha$ suppresses the contribution of a single quark state since the lower Dirac components of the quark wavefunctions are increasing. But it also reduces the quark state energy, so we have more states to sum over.

The main results of this paper can be summarized as follows: 1) Strange quark polarization is a very clean and robust prediction of the chiral potential model. Up to second order the only contribution arises from the diagram of Fig. 1. $\Delta s$ depends only weakly on the model parameters, and our calculation shows further that the variation of these parameters does not influence $\Delta s$ too much, provided we sum over the intermediate quark state up to a given energy. 2) The contribution from the intermediate excited quark states are important. It is not enough to restrict the intermediate state to the ground or the first few states. 3) Among the time-ordered diagrams, the quark-antiquark pair creation and annihilation " $Z$ " diagrams are significant. It is the " $Z$ " diagrams (Figs. $4 \mathrm{C}$ and $4 \mathrm{D}$ ) that introduce a negative value for $\Delta s$ in the nucleon, while the intermediate negative-energy states (Fig. 4B) gives a fairly large positive contribution. The importance of the pair creation and annihilation contribution to $\Delta q$ has also been noticed by some of us previously in a valence and sea quark mixing model [11]. 4) Our numerical result is quite consistent with experiments $\left(\Delta s\left(Q^{2}=3 \mathrm{GeV}^{2}\right)=-0.10 \pm 0.01 \pm \cdots\right.$, where the second \pm sign represents further sources of error, principally the low $x$ extrapolation [12]) and lattice QCD calculations $(\Delta s=-0.12(1) 13,-0.109(30)$ 14 $)$, and is also consistent with a schematic calculation in the context of chiral quark model by Cheng and $\mathrm{Li}$ [15]. To the best of our knowledge this is first time that $\Delta s$ is consistently calculated up to the one-loop level in a quark model.

This work is supported by the CNSF (19675018), CSED, CSSTC, the DFG (FA67/25-1), and the DAAD.

[1] For a review of the nucleon spin problem, see H.Y. Cheng, Int. J. Mod. Phys. A 11, 5109 (1996) hep-ph/9607254); R.L. Jaffe, Phys. Today 48(9), 24 (1995); U. Stiegler, Phys. Rep. 277, 1 (1996)

[2] T. Gutsche and D. Robson, Phys. Lett. B 229, 333 (1989).

[3] See, e.g.,A.W. Thomas, Advan. Nucl. Phys. 13, 1 (1984).

[4] G. Parisi, R. Petronzio, F. Rapuano, Phys. Lett. B 128, 418 (1983).

[5] J.D. Stack, Phys. Rev. D 29, 1213 (1984).

[6] M. Luscher, Nucl. Phys. B180, 317 (1981).

[7] S. Itoh, Y. Iwasaki, T. Yoshie, Phys. Rev. D 33, 1806 (1986).

[8] E. Eichten, K. Gottfried, T. Kinoshita, K.D. Lane, T.M. Yan, Phys. Rev. D 17, 3090 (1978); Phys. Rev. D 21, 203 (1980).

[9] G.S. Bali, K. Schilling, A. Wachter, Phys. Rev. D 56, 2566 (1997).

[10] X.S. Chen, X.B. Chen, A. Faessler, T. Gutsche, F. Wang, hep-ph/0005143.

[11] D. Qing, X.S. Chen, and F. Wang, Phys. Rev. C 57, R31 (1998); Phys. Rev. D 58, 114032 (1998).

[12] J. Ellis, hep-ph/9611208.

[13] S.J. Dong, J.P. Lagaë, and K.F. Liu, Phys. Rev. Lett. 75, 2096 (1995).

[14] M. Fukugita, Y. Kuramashi, M. Okawa, and A. Ukawa, Phys. Rev. Lett. 75, 2092 (1995).

[15] T.P. Cheng and L.F. Li, Phys. Rev. Lett. 74, 2872 (1995). 